\title{
Bernadette CUNNINGHAM, Medieval Irish Pilgrims to Santiago de Compostela
}

Delphine Planavergne

\section{(2) OpenEdition}

\section{Journals}

Édition électronique

URL : https://journals.openedition.org/ccm/8002

DOI : $10.4000 / \mathrm{ccm} .8002$

ISSN : 2119-1026

\section{Éditeur}

Centre d'études supérieures de civilisation médiévale/Université de Poitiers

\section{Édition imprimée}

Date de publication : 1 septembre 2021

Pagination : 256-259

ISBN : 978-2-490783-10-6

ISSN : 0007-9731

\section{Référence électronique}

Delphine Planavergne, "Bernadette cunningham, Medieval Irish Pilgrims to Santiago de Compostela », Cahiers de civilisation médiévale [En ligne], 255 | 2021, mis en ligne le 01 septembre 2021, consulté le 02 février 2022. URL : http://journals.openedition.org/ccm/8002 ; DOI : https://doi.org/10.4000/ccm. 8002

\section{(c) (i) (3)}

La revue Cahiers de civilisation médiévale est mise à disposition selon les termes de la Licence Creative Commons Attribution - Pas d'Utilisation Commerciale - Pas de Modification 4.0 International. 
Bernadette Cunningham, Medieval Irish Pilgrims to Santiago de Compostela, Dublin/Chicago, Four Courts Press, 2018.

Il y a un extraordinaire regain d'intérêt pour le pèlerinage de Saint-Jacques-de-Compostelle et dans cet ouvrage l'auteure, bibliothécaire adjointe à la bibliothèque de l'Académie royale irlandaise de Dublin, révèle qu'entre l'Irlande et le pèlerinage s'est noué un lien plus important que ce que l'on a longtemps pensé. L'histoire d'hommes et de femmes qui venaient d'Irlande pour SaintJacques-de-Compostelle au Moyen Âge témoigne de la participation irlandaise à un des pèlerinages majeurs de cette période. Le long et hasardeux voyage par terre et par mer vers les reliques de saint Jacques en Galice n'était pas entrepris à la légère. Ce livre novateur explore les diverses influences et les motivations des pèlerins aussi bien que la nature du voyage au Moyen Âge dans le but de comprendre quand, pourquoi et comment les pèlerins depuis l'Irlande allaient à Saint-Jacques à l'apogée du pèlerinage entre les $\mathrm{XII}^{\mathrm{e}}$ et $\mathrm{XV}^{\mathrm{e}} \mathrm{s}$. Il se fonde sur des documents officiels, des chroniques historiques, des textes littéraires, des Vies de saints et autres découvertes archéologiques pour découvrir ces pèlerins anglo-normands et gaéliques qui s'aventuraient 
depuis les confins de leurs propres communautés à la recherche du salut et peut-être d'un peu d'aventure. Après une introduction sur la modernité du pèlerinage multiséculaire, Bernadette Cunningham aborde son ouvrage en six chapitres : les motifs du pèlerinage médiéval (p. 17-43), le contexte du pèlerinage de Compostelle : la tradition du pèlerinage outre-mer depuis l'Irlande (p. 45-61), l'image de SaintJacques-de-Compostelle (p. 63-94), l'expérience du pèlerinage : la phase anglo-normande (p. 95-126), l'expérience du pèlerinage : la phase gaélo-irlandaise (p. 127-149), le déclin du pèlerinage médiéval et le regain de l'époque moderne (p. 151-163). Pour finir l'a. donne en appendices divers résultats de recherches sur les pèlerins à Saint Jacques depuis l'Irlande (appendice 1, p. 165-167), les églises irlandaises dédicacées à saint Jacques (appendice 2, p. 168-172), les biens consacrés à Jacques le Majeur (appendice 3, p. 173-174) et enfin les foires attestées lors de la saint Jacques (appendice 4, p. 175-176).

Dans le premier chapitre, l'a. se penche sur les raisons du pèlerinage médiéval. Un pèlerinage peut être défini par le fait d'entreprendre un voyage vers un lieu saint pour des motifs de dévotion dans le but d'obtenir une aide miraculeuse ou comme un acte de pénitence ou de remerciement. À partir de cette définition, elle montre que Saint-Jacques, devient au $\mathrm{XII}^{\mathrm{e}} \mathrm{s}$. un lieu de pèlerinage qui peut rivaliser avec ceux de Rome ou de Jérusalem pour les destinations à la suite de la rédaction de l'histoire de la découverte miraculeuse du tombeau du saint par un certain Pélage au $\mathrm{IX}^{\mathrm{e}} \mathrm{s}$. Cette histoire est rédigée dans un ouvrage fautivement attribué au pape Calixte II (1121-1124), mais dès lors le pèlerinage est lancé; les pèlerins vont se rendre auprès du tombeau et du reliquaire d'un des apôtres préférés du Christ comme le relatent les Évangiles et les Actes des Apôtres, ce qui en fait un intercesseur privilégié à prier.

Ensuite dans son deuxième chapitre, B. Cunningham nous fait part de la tradition des pèlerinages outre-mer en Irlande. C'est d'abord la tradition de la peregrinatio pro Deo ou pro Christo attestée depuis le $\mathrm{VII}^{\mathrm{e}} \mathrm{s}$. en Irlande; il s'agit d'un exil volontaire et permanent vécu comme un renoncement au monde, on devient un « exilé de Dieu » (p. 45), la séparation du monde qu'il connaît transforme le saint homme en une sorte de communicateur de l'autorité divine à l'image de saint Colomban le plus connu de ces peregrini, qui vint s'établir à Luxeuil puis ailleurs en
Gaule avant de fonder une communauté influente à Bobbio. Les propres mots du Christ « prends ta croix, et suis -moi » (Matt. $10: 8$ ) sont d'abord interprétés comme une invitation à vivre selon les préceptes du Christ, mais aussi à suivre les pas mêmes du Christ, c'est-à-dire à se rendre en Terre Sainte ce que certains Irlandais semblent avoir accompli entre le XII et le XIV $\mathrm{s}$. Mais face aux difficultés du voyage, surtout durant les croisades d'autres pèlerinages sont considérés comme des alternatives à ce qui est perçu comme le sommet des pèlerinages à savoir la Terre Sainte; il s'agit du pèlerinage de Rome et de celui de Saint-Jacques-de-Compostelle. Il y a donc en Irlande une forte tradition de pèlerinages outre-mer en Europe et au Proche-Orient; mais à partir du $\mathrm{XII}^{\mathrm{e}} \mathrm{s}$. celui de Saint-Jacques est beaucoup plus connu, Rome et Jérusalem continuent à être pratiqués, et celui de Rome devient de plus en plus banal après 1300 . Toutefois le nombre des Irlandais connus pour avoir atteint Rome ou Jérusalem est probablement très bas; comment et pourquoi le pèlerinage de Saint-Jacquesde-Compostelle se développe comme une alternative aux précédents à l'intérieur du monde chrétien et comment les Irlandais ont été amenés à le connaître sont les thèmes abordés dans le chapitre suivant.

Pour ceux qui s'engagent dans un pèlerinage, il s'agit d'un acte des plus rationnels; il est fondé comme une compréhension de l'économie du salut à la fois profonde et pragmatique. L'idée qu'entreprendre un voyage vers un lieu saint pourrait transformer quelqu'un pour toujours et dans l'au-delà, or celle-ci est très attractive et ceci malgré la connaissance des dangers que représente un voyage vers des lieux lointains. Mais comment connaît-on Saint-Jacquesde-Compostelle comme lieu de pèlerinage? Par des sermons au sujet du saint dont le reliquaire est montré, des récits racontés par les pèlerins revenus et qui ont entrepris le voyage et d'autres sources moindres. Le chemin le plus commun pour avoir une connaissance des traditions liées à Compostelle se retrouve dans les récits concernant Jacques le Majeur. Ces récits circulaient à la fois oralement et sous forme écrite et autour d'eux ont été construits d'autres éléments de narration. Il est difficile en effet d'éviter saint Jacques, à la fois présent dans les textes liturgiques et dans de nombreux contextes plus informels. Dans le Nouveau Testament, il est présenté comme le frère aîné de Jean, le rédacteur du quatrième évangile et comme fils de Zébédée; mais surtout avec son frère 
et saint Pierre, selon l'Évangile de Marc, ils sont les seuls à être présents lors de la résurrection de la fille de Jaïre (Marc 5 :35-43), de la Transfiguration (Marc 9 :2-13) et à accompagner Jésus au jardin de Gethsemani peu avant la trahison de Judas et la crucifixion (Matt. 26 :37). De fait les liens entre saint Jacques et l'Irlande ont été noués tôt au XII $\mathrm{s}$., lorsque l'Église d'Irlande a reconnu le saint dans son canon des saints. Mais la grande promotion du saint et de son pèlerinage est due à Jacques de Voragine qui se fonde pour écrire la Vita de Jacques le Majeur dans sa Légende dorée sur le récit rapporté par le Codex calixtinus, la collection la plus éminente de la propagande médiévale au sujet du tombeau de saint Jacques en Galice. Mais aussi des textes anciens, $\mathrm{VII}^{\mathrm{e}} \mathrm{s}$., montrent le lien privilégié de l'Irlande et l'Espagne lorsque le fils de Mil, ancêtre des Gaels, vient s'installer en Irlande depuis l'Égypte via le nord de la péninsule hispanique. Certes le culte de saint Jacques en Irlande ne peut rivaliser avec ceux de saints locaux comme Patrick, Brigitte ou Colum Cille. Mais les liens entre saint Jacques, le nord de la Galice et l'Irlande sont bien attestés au point que le pèlerinage devient un voyage obligé ne serait-ce que par les indulgences accordées au bout du compte; les conditions pour que les Irlandais soient alors réunis pour qu'ils rejoignent les hommes et les femmes du reste de l'Europe dans leur périple vers le sacré. Le culte est évident dans les régions anglo-saxonnes dès le $\mathrm{XIII}^{\mathrm{e}} \mathrm{s}$. mais devient des plus populaire chez les Gaéliques au $\mathrm{Xv}^{\mathrm{e}} \mathrm{s}$.

Il y a deux phases distinctes pour les pèlerins irlandais de Saint-Jacques. La première est dominée par l'influence anglo-normande pour laquelle les plus anciennes sources datent du XIII' ${ }^{\mathrm{e}} \mathrm{s}$. Ce n'est qu'à partir $\mathrm{du} X \mathrm{Xv}^{\mathrm{e}} \mathrm{s}$. que les pèlerins venus des parties gaéliques de l'Irlande sont intéressés pour partir vers Santiago. L'enthousiasme pour le culte de Jacques le Majeur de la part des colons anglo-normands d'Irlande n'est pas en relation directe avec le tombeau de Galice mais avec l'abbaye de Reading située dans le sud de l'Angleterre et qui conserve la main du saint, main réputée avoir opéré de nombreux miracles. Elle aurait été donnée par le roi Henri Ir en 1133 et lorsque l'épiscopat anglais et gallois a autorisé les indulgences pour un pèlerinage vers la relique en 1155, Reading est devenu le plus populaire des pèlerinages vers l'Angleterre jusqu'à la canonisation de Thomas Becket en 1173 et que Canterbury devint une alternative à cette destination. Reading fut le lieu de nombreux récits de miracles de guérisons attribués à saint Jacques; parmi celles-ci un miracle concernant un des jeunes hommes de la suite du prince Jean, fils du roi Henri II, qui se rendait en Irlande. Le jeune homme s'étant cassé le bras, il fit le vœu à saint Jacques d'aller à Reading en pèlerinage s'il recouvrait l'utilisation dudit bras; mais ayant négligé son vœu après sa guérison, il se cassa l'autre bras. Plein de remords, il fit confectionner un bras et une main en cire et les offrit au saint à Reading. Dès lors la promotion du pèlerinage fut très active en Irlande. Mais pour quelques fidèles de saint Jacques et une partie de l'élite anglaise et anglo-irlandaise, Reading ne suffisait pas et préférèrent Saint-Jacques de Galice comme destination de pèlerinage depuis l'Irlande et destination qui crut tout au long du XIII ${ }^{\mathrm{e}} \mathrm{s}$. avec des infrastructures et des voyages organisés dans ce but. Les plus anciens pèlerins sont des clercs et des officiers du gouvernement anglo-normands dont la carrière induit des déplacements et une extrême mobilité. Puis au $\mathrm{XV}^{\mathrm{e}} \mathrm{s}$., ce sont des citadins hommes et femmes relativement aisés qui entreprennent le voyage.

La seconde grande phase pour les pèlerins irlandais de Santiago peut être qualifiée de gaélique et se situe $\mathrm{au} \mathrm{XV} \mathrm{e}$. ; ce dernier connaît à ce moment-là un véritable «boom» (p. 147) ceci est à mettre en relation avec les indulgences plénières qui sont accordées aux pèlerins se rendant en Galice auprès de saint Jacques. C'est alors l'apogée du pèlerinage irlandais vers ce lieu lorsque les informations sont beaucoup plus nombreuses et que le voyage est plus facile à faire.

Avec la Réforme, pèlerinages et culte des reliques sont perçus comme une idolâtrie, de fait à partir du $\mathrm{XVI}^{\mathrm{e}} \mathrm{s}$. les pèlerinages entrent dans une phase de déclin, accentuée encore au moment des guerres de religion du XVII ${ }^{e}$ s. qui affectent les déplacements et découragent les pèlerins potentiels d'entreprendre un long voyage loin de leur patrie. Et l'Irlande ne fait pas exception à ce déclin. Il faut attendre la fin du XIX $\mathrm{s}$. pour voir un renouveau du pèlerinage au moment où les reliques de saint Jacques sont déclarées authentiques par le pape Léon XIII en 1884. Malgré un recul lors des deux guerres mondiales, Santiago connaît un renouveau à l'époque de Franco dans le cadre de son mouvement du catholicisme national. Dès lors le succès de la route de Compostelle ne se dément plus; toutefois ce n'est plus tellement la dévotion à Jacques le Majeur qu'une volonté de dépassement 
physique de soi, de voir de nouveau horizons et de rencontrer de nouveaux personnages.

Dans ce livre l'a. dresse un tableau très complet du pèlerinage de saint Jacques le Majeur à Compostelle. Sa méthode qui est fondée sur une analyse systématique des sources disponibles sur le sujet en fait un ouvrage sérieux et des plus documentés sur la question du pèlerinage irlandais de Santiago. D'une lecture aisée facilitée par la présence de conclusions partielles à chaque fin de chapitre cet ouvrage allie sérieux sur le fond et la documentation, et plaisir de la lecture sur un sujet somme toute assez novateur.

Delphine Planavergne Université Toulouse Jean Jaurès 